\title{
THE ELECTRON DENSITY IS SMOOTH AWAY FROM THE NUCLEI
}

\author{
SØREN FOURNAIS, MARIA HOFFMANN-OSTENHOF, THOMAS \\ HOFFMANN-OSTENHOF AND THOMAS ØSTERGAARD SØRENSEN
}

Abstract. We prove that the electron densities of electronic eigenfunctions of atoms and molecules are smooth away from the nuclei.

\section{Introduction and Statement of the Results.}

We consider an $N$-electron molecule with $L$ fixed nuclei whose nonrelativistic Hamiltonian is given by

$$
\begin{aligned}
H_{N, L}(\mathbf{R}, \mathbf{Z})=\sum_{j=1}^{N}( & \left.-\Delta_{j}-\sum_{l=1}^{L} \frac{Z_{l}}{\left|x_{j}-R_{l}\right|}\right) \\
& +\sum_{1 \leq i<j \leq N} \frac{1}{\left|x_{i}-x_{j}\right|}+\sum_{1 \leq l<k \leq L} \frac{Z_{l} Z_{k}}{\left|R_{l}-R_{k}\right|}
\end{aligned}
$$

where $\mathbf{R}=\left(R_{1}, R_{2}, \ldots, R_{L}\right) \in \mathbb{R}^{3 L}, R_{l} \neq R_{k}$ for $k \neq l$, denote the positions of the $L$ nuclei whose positive charges are given by $\mathbf{Z}=$ $\left(Z_{1}, Z_{2}, \ldots, Z_{L}\right)$. The positions of the $N$ electrons are denoted by $\left(x_{1}, x_{2}, \ldots, x_{N}\right) \in \mathbb{R}^{3 N}$ where $x_{j}$ denotes the position of the $j$-th electron in $\mathbb{R}^{3}$ and $\Delta=\sum_{j=1}^{N} \Delta_{j}$ is the $3 N$-dimensional Laplacian.

The operator $H_{N, L}(\mathbf{R}, \mathbf{Z})$ depends parametrically on $\mathbf{R}, \mathbf{Z}, L, N$ and the last term in (1.1) corresponds to the internuclear repulsion which is just an additive term. It will play no role in this paper and we will hence neglect it, i.e. from now on we let

$$
H=H_{N, L}(\mathbf{R}, \mathbf{Z})-\text { internuclear repulsion. }
$$

The operator $H$ is selfadjoint on $L^{2}\left(\mathbb{R}^{3 N}\right)$ with operator domain $\mathcal{D}(H)=W^{2,2}\left(\mathbb{R}^{3 N}\right)$ and quadratic form domain $\mathcal{Q}(H)=W^{1,2}\left(\mathbb{R}^{3 N}\right)$, see e.g. Kato $[8]$.

Date: October 27, 2018.

Work supported by the Carlsberg Foundation, European Union TMR grant FMRX-CT 96-0001, Ministerium für Wissenschaft und Verkehr der Republik Österreich, and the Austrian Science Foundation, grant number P12864-MAT. 
2 S. FOURNAIS, M. AND T. HOFFMANN-OSTENHOF AND T. Ø. SØRENSEN

We consider the eigenvalue problem

$$
H \psi=(-\Delta+V) \psi=E \psi,
$$

where the potential $V$ is given by

$$
V=-\sum_{j=1}^{N} \sum_{l=1}^{L} \frac{Z_{l}}{\left|x_{j}-R_{l}\right|}+\sum_{1 \leq i<j \leq N} \frac{1}{\left|x_{i}-x_{j}\right|} .
$$

Of course the eigenfunction $\psi$ and the associated eigenvalue $E$ depend parametrically on $\mathbf{R}, \mathbf{Z}, L, N$. Since the potential $V$ has singularities for $x_{j}=R_{l}$ and for $x_{i}=x_{j}$ one cannot expect classical solutions. In 1957 Kato showed [7] that any local solution $\psi$ to (1.2) is locally Lipschitz, i.e.

$$
\psi \in C_{\mathrm{loc}}^{0}\left(\mathbb{R}^{3 N}\right) \text { and }|\nabla \psi| \in L_{\mathrm{loc}}^{\infty}\left(\mathbb{R}^{3 N}\right) .
$$

Kato also characterized the behaviour of such a solution near points where two particles are close to each other (Cusp conditions). (Extensions of these results on the regularity of $\psi$ can be found in M. and T. Hoffmann-Ostenhof and Stremnitzer [6] and M. and T. HoffmannOstenhof and Østergaard Sørensen [5]). Of course, away from the singularities of $V$, any local solution $\psi$ of (1.2) is smooth by elliptic regularity.

Equation (1.2) is a partial differential equation in $3 N$ variables and hence only certain one-electron cases can be solved analytically. (Oneelectron atoms and diatomic one-electron molecules with equal nuclear charges).

Most of the information about bound states of atoms and molecules that Chemists and Physicists want to know is encoded in equation (1.2). (Of course one can go beyond (1.2), for instance allow for nuclear motion, include relativistic corrections, etc.). Since the electrons are Fermions the physically relevant wavefunctions $\psi$ have to satisfy the Pauli principle. This amounts to requiring that $\psi$ transforms according to some specific irreducible representations of the symmetric group $\mathfrak{S}_{N}$. Our results will include this.

Already in the early times of quantum mechanics in the 1920's and the 1930's various attempts were made to replace the full $3 N$-dimensional equation (1.2) by simpler (usually non-linear) ones in 3 dimensions (Thomas-Fermi theory and Hartree-Fock theory). The relation of these approximations with the N-electron Schrödinger equation has been analysed in detail for big atoms, see e.g. Lieb and Simon [10], Lieb [9], and Lieb and Simon 11.

One important observation and motivation for the development of these and other approximation schemes was the insight that in order to 
calculate the energy $E$ or one- and two-electron operator expectation values there is no need for the full wave function $\psi\left(x_{1}, x_{2}, \ldots, x_{N}\right)$ but only for the one-electron density $\rho(x), x \in \mathbb{R}^{3}$, the two-electron density $\rho_{2}\left(x, x^{\prime}\right),\left(x, x^{\prime}\right) \in \mathbb{R}^{6}$ and for the one-electron density matrix $\gamma_{1}\left(x, x^{\prime}\right),\left(x, x^{\prime}\right) \in \mathbb{R}^{6}$. These quantities are defined as follows: Defining

$$
\psi_{j}(x)=\psi\left(x_{1}, \ldots, x_{j-1}, x, x_{j+1}, \ldots, x_{N}\right)
$$

and

$$
\psi_{i, j}\left(x, x^{\prime}\right)=\psi\left(x_{1}, \ldots, x_{i-1}, x, x_{i+1}, \ldots, x_{j-1}, x^{\prime}, x_{j+1}, \ldots, x_{N}\right),
$$

and assuming without loss of generality that $\psi$ is real valued (since the coefficients of $H$ are real), the functions $\rho, \rho_{2}$, and $\gamma_{1}$ are given by

$$
\begin{aligned}
\rho(x) & =\sum_{j=1}^{N} \int \psi_{j}^{2} d \hat{x}_{j}, \\
\rho_{2}\left(x, x^{\prime}\right) & =\sum_{1 \leq i \neq j \leq N} \int \psi_{i, j}^{2} d \hat{x}_{i, j},
\end{aligned}
$$

and

$$
\gamma_{1}\left(x, x^{\prime}\right)=\sum_{j=1}^{N} \int \psi_{j}(x) \psi_{j}\left(x^{\prime}\right) d \hat{x}_{j} .
$$

Here $d \hat{x}_{j}$, respectively $d \hat{x}_{i, j}$, means integration over all variables except $x_{j}$, repectively $x_{i}, x_{j}$. Of course $\rho(x)=\gamma_{1}(x, x)$.

More recently very successful approximation schemes have been developped called Density Functional Theories (DFT). These schemes use some non-linear functionals in which only the one-electron density $\rho$ occurs and lead to surprisingly good approximations to ground state energies and molecular geometries (see e.g. Eschrig [1]). However, their relation to the full Schrödinger equation remains unclear.

It is therefore surprising that the eigenfunction $\psi$ and in particular the electron density $\rho$ itself, defined in (1.4), have only rarely been the subject of mathematical analysis (see [5] and references therein).

Here we prove, based on recent work [5], natural smoothness results about the quantities defined in (1.4), (1.5), and (1.6).

We do not assume anything about the eigenvalue $E$-in particular, it could be an embedded eigenvalue. The only assumption is that $\psi$ satisfies the following decay estimate:

$$
|\psi(\mathbf{x})| \leq c e^{-\lambda|\mathbf{x}|} \text { for all } \mathbf{x} \in \mathbb{R}^{3 N},
$$


4 S. FOURNAIS, M. AND T. HOFFMANN-OSTENHOF AND T. Ø. SøRENSEN

for some $c, \lambda>0$.

Remark 1.1. Since $\psi$ is continuous, (1.7) is only an assumption on the behaviour near infinity. For references on the exponential decay of eigenfunctions, see e.g. Simon [12].

Remark 1.2. We only assume the exponential decay for simplicity. Our proofs extend to the case where the eigenfunction $\psi$ decays faster than polynomially.

The main result of this paper is the following theorem:

Theorem 1.3. Let $\psi$ be an eigenfunction of $H$, satisfying (1.X). Let $\rho, \rho_{2}$, and $\gamma_{1}$ be as defined in (1.4), (1.5), and (1.6), and define furthermore $n(x)=\rho_{2}(x, x)$.

Let $D=\left\{(x, x) \in \mathbb{R}^{6}\right\} \subset \mathbb{R}^{6}$, and define

$$
\Sigma=\left(\left\{R_{1}, \ldots, R_{L}\right\} \times \mathbb{R}^{3}\right) \cup\left(\mathbb{R}^{3} \times\left\{R_{1}, \ldots, R_{L}\right\}\right) \subset \mathbb{R}^{6} .
$$

Then

$$
\rho, n \in C^{\infty}\left(\mathbb{R}^{3} \backslash\left\{R_{1}, \ldots, R_{L}\right\}\right)
$$

and

$$
\rho_{2} \in C^{\infty}\left(\mathbb{R}^{6} \backslash(\Sigma \cup D)\right), \gamma_{1} \in C^{\infty}\left(\mathbb{R}^{6} \backslash \Sigma\right) .
$$

Furthermore, all the derivatives of $\rho$ satisfy an exponential decay estimate near infinity: Let $\gamma \in \mathbb{N}^{3}$ be a multi-index. Then for all $\epsilon>0$ there exists a constant $c=c(\gamma, \epsilon)$ such that

$$
\left|\partial_{x}^{\gamma} \rho(x)\right| \leq c e^{-(\lambda-\epsilon)|x|} \quad \text { for all } \quad|x|>\max \left\{\left|R_{1}\right|, \ldots,\left|R_{L}\right|\right\}+1 .
$$

Similar results hold for $n$-electron densities and for $n$-electron density matrices.

Remark 1.4. For simplicity we will only prove Theorem 1.3 for atoms (i.e. for $L=1, R_{1}=0$ ) and only indicate the necessary modifications for the molecular case. Moreover, we only explicitly treat the density $\rho$, the proofs in the cases of $n, \rho_{2}$, and $\gamma_{1}$ being essentially the same. Finally, it is enough to prove smoothness of each term $\int \psi_{j}^{2} d \hat{x}_{j}$ in the sum (1.4), and we therefore restrict ourselves to

$$
\int \psi_{1}^{2} d \hat{x}_{1}=\int \psi^{2}\left(x, x_{2}, \ldots, x_{N}\right) d x_{2} \cdots d x_{N}
$$




\section{Additional Regularity of Eigenfunctions.}

We will need to know something about the regularity properties of $\psi$ in order to conclude that $\rho$ is smooth. In order to study the regularity of $\psi$ it is convenient to work in spaces of functions which are Hölder continuous. Let us recall the definition of Hölder continuity:

Definition 2.1. Let $\Omega$ be a domain in $\mathbb{R}^{n}, k \in \mathbb{N}$, and $\alpha \in(0,1]$. We say that a function $u$ belongs to $C^{k, \alpha}(\Omega)$ whenever $u \in C^{k}(\Omega)$, and for all $\beta \in \mathbb{N}^{n}$ with $|\beta|=k$, and all open balls $B\left(x_{0}, r\right)$ with $\overline{B\left(x_{0}, r\right)} \subset \Omega$, we have

$$
\sup _{x, y \in B\left(x_{0}, r\right), x \neq y} \frac{\left|\partial^{\beta} u(x)-\partial^{\beta} u(y)\right|}{|x-y|^{\alpha}} \leq C\left(x_{0}, r\right) .
$$

For any domain $\Omega^{\prime}$, with $\overline{\Omega^{\prime}} \subset \Omega$, we now define the following norms:

$$
|u|_{C^{k, \alpha}\left(\Omega^{\prime}\right)}=\sum_{|\beta| \leq k}\left\|\partial^{\beta} u\right\|_{L^{\infty}\left(\Omega^{\prime}\right)}+\sum_{|\beta|=k} \sup _{x, y \in \Omega^{\prime}, x \neq y} \frac{\left|\partial^{\beta} u(x)-\partial^{\beta} u(y)\right|}{|x-y|^{\alpha}} .
$$

We will need the following result on elliptic regularity in order to conclude that the solutions of elliptic second order equations with bounded coefficients are $C^{1, \alpha}$. The proposition is a reformulation of Corollary 8.12 in Gilbarg and Trudinger [4], adapted for our purposes:

Proposition 2.2. Let $\Omega$ be a bounded domain in $\mathbb{R}^{n}$ and suppose $u \in$ $W^{1,2}(\Omega)$ is a weak solution of $\Delta u+\sum_{j=1}^{n} b_{j} D_{j} u+W u=g$ in $\Omega$, where $b_{j}, W, g \in L^{\infty}(\Omega)$. Then $u \in C^{1, \alpha}(\Omega)$ for all $\alpha \in(0,1)$ and for any domain $\Omega^{\prime}, \overline{\Omega^{\prime}} \subset \Omega$ we have

$$
|u|_{C^{1, \alpha}\left(\Omega^{\prime}\right)} \leq C\left(\sup _{\Omega}|u|+\sup _{\Omega}|g|\right)
$$

for $C=C\left(n, M, \operatorname{dist}\left(\Omega^{\prime}, \partial \Omega\right)\right)$, with

$$
\max _{j=1, \ldots, n}\left\{1,\left\|b_{j}\right\|_{L^{\infty}(\Omega)},\|W\|_{L^{\infty}(\Omega)},\|g\|_{L^{\infty}(\Omega)}\right\} \leq M
$$

Our regularity result on $\psi$ is the following:

Lemma 2.3. Let $\psi$ be an $N$-electron atomic eigenfunction satisfying the decay estimate (1.7). Let $P$ and $Q$ be a partition of $\{1, \ldots, N\}$ :

$$
\{1, \ldots, N\}=P \cup Q, \quad P \cap Q=\emptyset, \quad P \neq \emptyset .
$$

Define $x_{P}$ as

$$
x_{P}=\frac{1}{\sqrt{|P|}} \sum_{j \in P} x_{j} \in \mathbb{R}^{3},
$$

and let $T$ be any orthogonal transformation such that $T\left(x_{1}, \ldots, x_{N}\right)=$ $\left(x_{P}, x^{\prime}\right)$ with $x^{\prime} \in \mathbb{R}^{3 N-3}$. 
6 S. FOURNAIS, M. AND T. HOFFMANN-OSTENHOF AND T. Ø. SøRENSEN

Let $\epsilon>0$ be given and define $U_{P} \subset \mathbb{R}^{3 N}$ as the open set:

$$
\begin{aligned}
U_{P}=\left\{\left(x_{1}, \ldots, x_{N}\right) \in \mathbb{R}^{3 N} \mid\right. & \left|x_{j}\right|>\epsilon \text { for } j \in P \\
& \left.\left|x_{j}-x_{k}\right|>\epsilon \text { for } j \in P, k \in Q\right\} .
\end{aligned}
$$

Then

$$
\partial_{x_{P}}^{\gamma}\left(\psi \circ T^{*}\right) \in C^{0,1}\left(T U_{P}\right) \quad \text { for all } \gamma \in \mathbb{N}^{3} .
$$

Furthermore, the following a priori estimate holds:

$$
\begin{aligned}
\left|\nabla \partial_{x_{P}}^{\gamma}\left(\psi \circ T^{*}\right)\left(x_{P}, x^{\prime}\right)\right|+\mid \partial_{x_{P}}^{\gamma}( & \left.\psi \circ T^{*}\right)\left(x_{P}, x^{\prime}\right) \mid \\
& \leq C e^{-\lambda\left|\left(x_{P}, x^{\prime}\right)\right|} \quad \text { for all }\left(x_{P}, x^{\prime}\right) \in T U_{P}
\end{aligned}
$$

for some $C=C(\gamma)>0$.

Remark 2.4. One could interpret $U_{P}$ as a (very large) neighbourhood of a singularity $\mathbf{x}^{0}$ of $V$ where the electrons with coordinates $x_{j}$ with $j \in Q$ sit on the nucleus and the electrons with coordinates $x_{j}$ with $j \in P$ sit on each other away from the nucleus, i.e. $\mathbf{x}^{0}=\left(x_{1}^{0}, \cdots, x_{N}^{0}\right)$, with

$$
\begin{aligned}
x_{j}^{0}=0 & \text { for all } j \in Q, \\
x_{j}^{0}=y^{0} \neq 0 & \text { for all } j \in P .
\end{aligned}
$$

Notice that $x_{P}$ is (up to a scalar multiple) the centre of mass of the electrons $j$ with $j \in P$. Loosely speaking Lemma 2.3 then says that in the neighbourhood $U_{P}, \psi$ is smooth with respect to the centre of mass coordinate $x_{P}$.

One can also consider $\mathbf{x}^{0}$ as a two-cluster singularity-one group of electrons on each other at the nucleus, another group of electrons on top of each other away from the nucleus. It is, of course, possible to have many clusters. Lemma 2.3 is a special case of a more general theorem: If we have any number of clusters which are separated from each other and from the nucleus, then the eigenfunction $\psi$ can be differentiated any number of times with respect to the centre of mass of each cluster.

We only need the two-cluster version (see Lemma 2.3) in the present paper. The more general result will be used in a detailed investigation of the regularity of the wavefunction near all kinds of singularities of the potential (see [3]). 
THE ELECTRON DENSITY IS SMOOTH AWAY FROM THE NUCLEI 7

Remark 2.5. In the case of molecules the definition of $U_{P}$ is slightly different:

$$
\begin{array}{r}
U_{\mathrm{MOL}}=\left\{\left(x_{1}, \ldots, x_{N}\right) \in \mathbb{R}^{3 N}\left|\min _{1 \leq l \leq L}\right| x_{j}-R_{l} \mid>\epsilon \text { for } j \in P,\right. \\
\left.\left|x_{j}-x_{k}\right|>\epsilon \text { for } j \in P, k \in Q\right\} .
\end{array}
$$

Apart from that, Lemma 2.3 remains unchanged.

Before we prove Lemma 2.3, let us fix some notation. We may assume without loss of generality that $P=\left\{1, \ldots, N_{1}\right\}$, with $N_{1} \leq N$. Then any orthogonal transformation $T$ satisfying the assumptions in the statement of Lemma 2.3 can be written as:

$$
T=\left(\begin{array}{cccccc}
\frac{1}{\sqrt{N_{1}}} & \cdots & \frac{1}{\sqrt{N_{1}}} & 0 & \cdots & 0 \\
& & & & & \\
& & \tilde{T} & & \\
& & & & &
\end{array}\right),
$$

with the first row being understood as $3 \times 3$ matrices - first $N_{1}$ repetitions of $\frac{1}{\sqrt{N_{1}}} I_{3}$ and then $N-N_{1}$ repetitions of the $3 \times 30$-matrix. The remaining part of the matrix, $\tilde{T} \in M_{3 N-3,3 N}(\mathbb{R})$ is such that the complete matrix $T$ is orthogonal. We will denote the $(3 N-3) \times 3$-columns of $\tilde{T}$ by $t_{j}$, i.e.

$$
\tilde{T}=\left(\begin{array}{lll}
t_{1} & \cdots & t_{N}
\end{array}\right),
$$

with $t_{j} \in M_{3 N-3,3}(\mathbb{R})$. Then we get:

$$
T^{*}=\left(\begin{array}{cc}
\frac{1}{\sqrt{N_{1}}} & t_{1}^{*} \\
\vdots & \vdots \\
\frac{1}{\sqrt{N_{1}}} & t_{N_{1}}^{*} \\
0 & t_{N_{1}+1}^{*} \\
\vdots & \vdots \\
0 & t_{N}^{*}
\end{array}\right) .
$$

Proof. For the proof of Lemma 2.3 we first proceed as in M. and T. Hoffmann-Ostenhof and Østergaard Sørensen [5]: We make the 'Ansatz'

$$
\psi=e^{F-F_{1}} \psi_{1},
$$


8 S. FOURNAIS, M. AND T. HOFFMANN-OSTENHOF AND T. $\varnothing$. SØRENSEN

with

$$
F=\sum_{j=1}^{N}-\frac{Z}{2}\left|x_{j}\right|+\sum_{1 \leq j<k \leq N} \frac{1}{4}\left|x_{j}-x_{k}\right|,
$$

and

$$
F_{1}=\sum_{j=1}^{N}-\frac{Z}{2} \sqrt{\left|x_{j}\right|^{2}+1}+\sum_{1 \leq j<k \leq N} \frac{1}{4} \sqrt{\left|x_{j}-x_{k}\right|^{2}+1} .
$$

Observing (see (1.3) with $L=1, R_{1}=0$ ),

$$
\Delta F=V \text {, }
$$

we get the following equation for $\psi_{1}$ using (1.2) and (2.3):

$$
\Delta \psi_{1}+2 \nabla\left(F-F_{1}\right) \cdot \nabla \psi_{1}+\left(\left|\nabla\left(F-F_{1}\right)\right|^{2}-\Delta F_{1}+E\right) \psi_{1}=0
$$

Furthermore, $F_{1}$ has the same behaviour as $F$ at infinity, so we get the following estimates:

$$
\begin{aligned}
\left\|\partial^{\beta} F_{1}\right\|_{L^{\infty}\left(\mathbb{R}^{3 N}\right)},\left\|F-F_{1}\right\|_{L^{\infty}\left(\mathbb{R}^{3 N}\right)}, & \left\|\nabla\left(F-F_{1}\right)\right\|_{L^{\infty}\left(\mathbb{R}^{3 N}\right)} \\
& \leq C(N, Z, \beta), \beta \in \mathbb{N}^{3 N},|\beta|>0 .
\end{aligned}
$$

We will first investigate the necessary regularity properties of the prefactor $e^{F-F_{1}} \circ T^{*}$ in $T U_{P}$ with respect to $x_{P}$. Then we will differentiate the equation (2.5) with respect to $x_{P}$ and analyse the regularity of $\partial_{x_{P}}^{\gamma}\left(\psi_{1} \circ T^{*}\right)$ in $T U_{P}$.

In the coordinates defined by $T$ we have

$$
\begin{array}{ll}
x_{j}=\frac{x_{P}}{\sqrt{N_{1}}}+t_{j}^{*} x^{\prime} & \text { for } j \leq N_{1}, \\
x_{j}=t_{j}^{*} x^{\prime} & \text { for } j>N_{1} .
\end{array}
$$

So

$$
x_{j}-x_{k}=\left(t_{j}^{*}-t_{k}^{*}\right) x^{\prime} \text { for } j, k \leq N_{1} \text { or } j, k>N_{1} .
$$

In particular these last expressions are independent of $x_{P}$. So when we differentiate $F \circ T^{*}$ with respect to $x_{P}$, the only non-vanishing terms come from derivatives of $\left|x_{j}\right| \circ T^{*}$ with $j \leq N_{1}$ and $\left|x_{j}-x_{k}\right| \circ T^{*}$ with $j \leq N_{1}, k>N_{1}$. The definition of $U_{P}$ implies that the function $\mathbf{x} \mapsto\left|x_{j}\right|$ is smooth with bounded derivatives on $U_{P}$ for $j \leq N_{1}$, and $\mathbf{x} \mapsto\left|x_{j}-x_{k}\right|$ is smooth with bounded derivatives on $U_{P}$ for $j \leq N_{1}$, $k>N_{1}$. Thus

$$
\begin{aligned}
\left\|\nabla \partial_{x_{P}}^{\gamma}\left(F-F_{1}\right) \circ T^{*}\right\|_{L^{\infty}\left(T U_{P}\right)} & +\left\|\partial_{x_{P}}^{\gamma}\left(F-F_{1}\right) \circ T^{*}\right\|_{L^{\infty}\left(T U_{P}\right)} \\
& \leq C(\gamma) \text { for all } \gamma \in \mathbb{N}^{3} .
\end{aligned}
$$


Furthermore, we get using (2.6) that

$$
\partial_{x_{P}}^{\gamma}\left(e^{F-F_{1}} \circ T^{*}\right) \in C^{0,1}\left(T U_{P}\right) \cap L^{\infty}\left(T U_{P}\right) \text { for all } \gamma \in \mathbb{N}^{3} .
$$

Hence, due to (2.3) and (2.9), it remains to study the regularity of $\psi_{1} \circ T^{*}$.

In the rest of the proof we will use the following notation: We will write $\tilde{F}, \tilde{F}_{1}, \tilde{\psi}_{1}$ instead of $F \circ T^{*}, F_{1} \circ T^{*}, \psi_{1} \circ T^{*}$. In particular we have the following relation

$$
\psi \circ T^{*}=e^{\tilde{F}-\tilde{F}_{1}} \tilde{\psi}_{1} .
$$

Notice that since the Laplacian is invariant under orthogonal transformations we have the following equation for $\psi \circ T^{*}$ :

$$
-\Delta\left(\psi \circ T^{*}\right)+\left(V \circ T^{*}\right)\left(\psi \circ T^{*}\right)=E\left(\psi \circ T^{*}\right) .
$$

Now, once again using the invariance of the Laplacian and (2.4),

$$
\Delta \tilde{F}=V \circ T^{*}
$$

so we get the following equation for $\tilde{\psi}_{1}$ (compare with (2.5)):

$$
\begin{aligned}
& L \tilde{\psi}_{1}=0, \\
& L=\Delta+2 \nabla\left(\tilde{F}-\tilde{F}_{1}\right) \cdot \nabla+\left(\left|\nabla\left(\tilde{F}-\tilde{F}_{1}\right)\right|^{2}-\Delta \tilde{F}_{1}+E\right) .
\end{aligned}
$$

The analysis of $\tilde{\psi}_{1}$ will be based on the elliptic regularity result from Proposition 2.2. We shall proceed by induction and for this we will need the following open sets indexed by $l \in \mathbb{N}$ :

$$
\begin{aligned}
U_{l}=\left\{\left(x_{1}, \ldots, x_{N}\right) \in \mathbb{R}^{3 N} \mid\right. & \left|x_{j}\right|>\epsilon\left(1-2^{-(l+1)}\right) \text { for } j \in P, \\
& \left.\left|x_{j}-x_{k}\right|>\epsilon\left(1-2^{-(l+1)}\right) \text { for } j \in P, k \in Q\right\} .
\end{aligned}
$$

It is clear that for $l_{1}<l_{2}$ we have

$$
U_{P} \subset U_{l_{2}} \subset U_{l_{1}} \subset U_{0} .
$$

We will prove the following statement:

Statement. For all $\gamma \in \mathbb{N}^{3}$ we have

(1) $\partial_{x_{P}}^{\gamma} \tilde{\psi}_{1} \in C^{1, \alpha}\left(T U_{|\gamma|}\right)$ for all $\alpha \in(0,1)$.

(2) $\partial_{x_{P}}^{\gamma} \tilde{\psi}_{1} \in W_{\text {loc }}^{2,2}\left(T U_{|\gamma|}\right)$.

(3) There exists $c=c(\gamma)>0$ such that

$$
\left|\nabla \partial_{x_{P}}^{\gamma} \tilde{\psi}_{1}\left(x_{P}, x^{\prime}\right)\right|+\left|\partial_{x_{P}}^{\gamma} \tilde{\psi}_{1}\left(x_{P}, x^{\prime}\right)\right| \leq c e^{-\lambda\left|\left(x_{P}, x^{\prime}\right)\right|}
$$

for all $\left(x_{P}, x^{\prime}\right) \in T U_{|\gamma|}$. 
10S. FOURNAIS, M. AND T. HOFFMANN-OSTENHOF AND T. Ø. SØRENSEN

Let us start by noticing that once this statement is established Lemma 2.3 is proved.

The proof of the statement proceeds by induction with respect to $|\gamma|$.

In order to prove the statement for $|\gamma|=0$, let us look at the equation (2.11). We get from Proposition 2.2 that

$$
\tilde{\psi}_{1} \in C^{1, \alpha}\left(T U_{0}\right)
$$

since the coefficients of the equation (2.11) are bounded on $T U_{0}$, due to (2.6). This proves (1) for $|\gamma|=0$.

We next prove that $\widetilde{\psi}_{1} \in W_{\text {loc }}^{2,2}\left(T U_{0}\right)$. This is accomplished as follows: If we use that $\tilde{\psi}_{1} \in C^{1, \alpha}\left(T U_{0}\right)$ and that the coefficients in the equation (2.11) (derivatives of $\tilde{F}-\tilde{F}_{1}$ ) are bounded (again using (2.6)), then it is easily seen from (2.11) that

$$
\Delta \tilde{\psi}_{1} \in L_{\mathrm{loc}}^{2}\left(T U_{0}\right)
$$

Therefrom, we get via standard elliptic regularity results (see for instance Folland [2, Theorem 6.33]) that $\tilde{\psi}_{1} \in W_{\text {loc }}^{2,2}\left(T U_{0}\right)$.

Next, we verify the exponential decay estimate (3) for $|\gamma|=0$. We know from the assumption (1.7) that $\tilde{\psi}_{1}$ decays exponentially, but we also need to prove it for $\nabla \tilde{\psi}_{1}$. This is done exactly as in the induction step below, using the exponential decay estimate (1.7). In order not to repeat the argument, we refer the reader to the induction step below.

Suppose now that we have proved (1)-(3) for all $\gamma$ with $|\gamma| \leq k$. Take a $\gamma$ with length $|\gamma|=k+1$.

Differentiating the equation (2.11) for $\tilde{\psi}_{1}$ we get the following equation for $\partial_{x_{P}}^{\gamma} \tilde{\psi}_{1}$ (in the sense of distributions):

$$
\begin{aligned}
& L\left(\partial_{x_{P}}^{\gamma} \tilde{\psi}_{1}\right)=f_{\gamma}, \\
& \begin{array}{l}
L=\Delta+2 \nabla\left(\tilde{F}-\tilde{F}_{1}\right) \cdot \nabla+\left(\left|\nabla\left(\tilde{F}-\tilde{F}_{1}\right)\right|^{2}-\Delta \tilde{F}_{1}+E\right) \\
f_{\gamma}=-\sum_{\sigma+\mu=\gamma,|\mu|<|\gamma|}\left(2\left[\partial_{x_{P}}^{\sigma}\left(\nabla\left(\tilde{F}-\tilde{F}_{1}\right)\right)\right] \cdot \nabla \partial_{x_{P}}^{\mu} \tilde{\psi}_{1}\right. \\
\left.\quad+\left[\partial_{x_{P}}^{\sigma}\left(\left|\nabla\left(\tilde{F}-\tilde{F}_{1}\right)\right|^{2}-\Delta \tilde{F}_{1}+E\right)\right] \partial_{x_{P}}^{\mu} \tilde{\psi}_{1}\right) .
\end{array}
\end{aligned}
$$

In $f_{\gamma}$ only partial derivatives of $\tilde{\psi}_{1}$ of length $\leq k$ occur.

It is clear that in (2.9) we may replace $T U_{P}$ by $T U_{0}$. Therefore we get, using the induction hypothesis for (1), that $f_{\gamma} \in L^{\infty}\left(T U_{|\gamma|-1}\right)$. A priori $\partial_{x_{P}}^{\gamma} \tilde{\psi}_{1}$ only satisfies the equation (2.12) in the distributional sense. In order to apply Proposition 2.2 we need that $\partial_{x_{P}}^{\gamma} \tilde{\psi}_{1} \in W_{\text {loc }}^{1,2}\left(U_{|\gamma|-1}\right)$. However, this follows from the induction hypotheses for (2), and the 
definition of Sobolev spaces. Now, due to (2.9), the coefficients of $L$ are bounded, and therefore we get from Proposition 2.2 that $\partial_{x_{P}}^{\gamma} \tilde{\psi}_{1} \in$ $C^{1, \alpha}\left(T U_{|\gamma|-1}\right)$ for all $\alpha \in(0,1)$, proving (1). This together with (2.9) implies that $f_{\gamma} \in L_{\text {loc }}^{2}\left(T U_{|\gamma|-1}\right)$. Therefore, we get (invoking once again that $\partial_{x_{P}}^{\gamma} \tilde{\psi}_{1} \in C^{1, \alpha}\left(T U_{|\gamma|-1}\right)$ for all $\alpha \in(0,1)$ and $\left.(2.9)\right)$,

$$
\Delta\left(\partial_{x_{P}}^{\gamma} \tilde{\psi}_{1}\right) \in L_{\mathrm{loc}}^{2}\left(T U_{|\gamma|-1}\right) .
$$

As before, via a standard elliptic regularity theorem (e.g. Folland [2, Lemma 6.32]), we see that $\partial_{x_{P}}^{\gamma} \tilde{\psi}_{1} \in W_{\text {loc }}^{2,2}\left(T U_{|\gamma|-1}\right)$, and therefore (2) is proved.

Finally, we prove the exponential decay of $\partial_{x_{P}}^{\gamma} \tilde{\psi}_{1}$. Let us write $\epsilon_{l}=$ $\epsilon / 2^{l+3}$. Then we have for all $\mathbf{x} \in U_{l}$ that $\overline{B\left(\mathbf{x}, 2 \epsilon_{l}\right)} \subset U_{l-1}$.

Since the equation (2.12) is satisfied on $T U_{|\gamma|-1}$ it is in particular satisfied on $T B\left(\mathbf{x}, 2 \epsilon_{|\gamma|}\right)$ for any $\mathbf{x} \in U_{|\gamma|}$. Applying Proposition 2.2 with $\Omega^{\prime}=T B\left(\mathbf{x}, \epsilon_{|\gamma|}\right), \Omega=T B\left(\mathbf{x}, 2 \epsilon_{|\gamma|}\right)$, we get that

$$
\left|\partial_{x_{P}}^{\sigma} \tilde{\psi}_{1}\right|_{C^{1, \alpha}\left(\Omega^{\prime}\right)} \leq C\left(\sup _{\Omega}\left|\partial_{x_{P}}^{\sigma} \tilde{\psi}_{1}\right|+\sup _{\Omega}\left|f_{\sigma}\right|\right) .
$$

Notice, using (2.9), that the coefficients in the differential operator $L$ are uniformly bounded on all of $T U_{0}$. Furthermore, the derivatives of $\tilde{F}$ and $\tilde{F}_{1}$ in the expression for $f_{\gamma}$ are also uniformly bounded on $U_{0}$ once again using (2.9). Therefore, using the induction hypothesis (3), we get for all $\left(x_{P}, x^{\prime}\right) \in T U_{|\gamma|}$ :

$$
\begin{aligned}
\left|\nabla \partial_{x_{P}}^{\sigma} \tilde{\psi}_{1}\left(x_{P}, x^{\prime}\right)\right| & \leq\left|\partial_{x_{P}}^{\sigma} \tilde{\psi}_{1}\right|_{C^{1, \alpha}\left(\Omega^{\prime}\right)} \\
& \leq C\left(\sup _{\Omega}\left|\partial_{x_{P}}^{\sigma} \tilde{\psi}_{1}\right|+\sup _{\Omega}\left|f_{\sigma}\right|\right) \\
& \leq C \sup _{\left(y_{P}, y^{\prime}\right) \in T B\left(\left(x_{P}, x^{\prime}\right), 2 \epsilon_{|\gamma|}\right)} e^{-\lambda\left|\left(y_{P}, y^{\prime}\right)\right|} \\
& =C e^{-\lambda\left|\left(x_{P}, x^{\prime}\right)\right|} .
\end{aligned}
$$

In this last line the constant $C$ does not depend on the position $\left(x_{P}, x^{\prime}\right)$ of the ball, since the coefficients of the equation (2.12) are uniformly bounded on all $T U_{0}$. This proves (3) and thus finishes the induction.

Remark 2.6. In the case of molecules we modify $F$ and $F_{1}$ as follows:

$$
F=\sum_{l=1}^{L} \sum_{j=1}^{N}-\frac{Z_{l}}{2}\left|x_{j}-R_{l}\right|+\sum_{1 \leq j<k \leq N} \frac{1}{4}\left|x_{j}-x_{k}\right|,
$$


12S. FOURNAIS, M. AND T. HOFFMANN-OSTENHOF AND T. $\varnothing$. SØRENSEN

and

$$
F_{1}=\sum_{l=1}^{L} \sum_{j=1}^{N}-\frac{Z_{l}}{2} \sqrt{\left|x_{j}-R_{l}\right|^{2}+1}+\sum_{1 \leq j<k \leq N} \frac{1}{4} \sqrt{\left|x_{j}-x_{k}\right|^{2}+1} .
$$

The rest of the proof is analogous.

\section{The Proof of TheOrem 1.3}

As noted in Remark 1.4 it is enough to prove smoothness of each individual term in (1.4). We therefore redefine $\rho$ by

$$
\rho(x)=\int \psi^{2}\left(x, x_{2}, \ldots, x_{N}\right) d x_{2} \cdots d x_{N} .
$$

Lemma 2.3 will be essential in order to prove the smoothness of $\rho$. It suffices to prove that $\rho \in C^{\infty}\left(\mathbb{R}^{3} \backslash \overline{B(0, R)}\right)$ for all $R>0$. Therefore, let us assume that $|x|>R>0$.

Remark 3.1. In the case of molecules we assume

$$
\min _{1 \leq l \leq L}\left|x-R_{l}\right|>R>0
$$

and prove that $\rho \in C^{\infty}\left(\mathbb{R}^{3} \backslash\left(\cup_{l=1}^{L} \overline{B\left(R_{l}, R\right)}\right)\right)$.

Let $\chi_{1}, \chi_{2}$ be a partition of unity in $\mathbb{R}_{+}: \chi_{1}+\chi_{2}=1, \chi_{1}(x)=1$ on $[0, R /(4 N)]$, supp $\chi_{1} \subset[0, R /(2 N)]$ and $\chi_{j} \in C^{\infty}\left(\mathbb{R}_{+}\right)$for $j=1,2$.

We combine the $\chi_{j}$ 's to make a partition of unity in $\mathbb{R}^{3 N}$. Obviously:

$$
1=\prod_{1 \leq j<k \leq N}\left(\chi_{1}+\chi_{2}\right)\left(\left|x_{j}-x_{k}\right|\right)
$$

Multiplying out the above product, we get sums of products of $\chi_{1}$ 's and $\chi_{2}$ 's. We introduce the following index sets to control these sums: Define first

$$
M=\left\{(j, k) \in\{1, \ldots, N\}^{2} \mid j<k\right\}
$$

and let

$$
\begin{aligned}
& I \subset M, \\
& J=M \backslash I .
\end{aligned}
$$

Now define, for each pair $I, J$ as above,

$$
\phi_{I}(\mathbf{x})=\left(\prod_{(j, k) \in I} \chi_{1}\left(\left|x_{j}-x_{k}\right|\right)\right)\left(\prod_{(j, k) \in J} \chi_{2}\left(\left|x_{j}-x_{k}\right|\right)\right) .
$$


Then we get

$$
1=\prod_{1 \leq j<k \leq N}\left(\chi_{1}+\chi_{2}\right)\left(\left|x_{j}-x_{k}\right|\right)=\sum_{I \subset M} \phi_{I}(\mathbf{x}),
$$

where the sum is over all subsets $I \subset M$.

Therefore we obtain:

$$
\begin{aligned}
\rho\left(x_{1}\right) & =\int_{\mathbb{R}^{3(N-1)}} \psi^{2}\left(x_{1}, x_{2}, \ldots, x_{N}\right) d x_{2} \cdots d x_{N} \\
& =\sum_{I \subset M} \int \psi^{2}\left(x_{1}, x_{2}, \ldots, x_{N}\right) \phi_{I}\left(x_{1}, x_{2}, \ldots, x_{N}\right) d x_{2} \cdots d x_{N} \\
& \equiv \sum_{I \subset M} \rho_{I}\left(x_{1}\right) .
\end{aligned}
$$

We will prove smoothness of each of the individual $\rho_{I}$ 's, so let us pick and fix an arbitrary $I \subset M$. Our strategy is to associate to $I$ a subset $P$ of $\{1, \ldots, N\}$ such that Lemma 2.3 is applicable, i.e. such that $\operatorname{supp} \phi_{I} \subset U_{P}$ with $U_{P}$ defined in (2.1) with a suitable $\epsilon>0$.

Remark 3.2. To motivate the determination of $P$ and hence the coordinate $x_{P}$ with respect to which we are allowed to differentiate, let us consider the following example: Let $N=3$ and $I=\{(1,2),(2,3)\}$ and $J=\{(1,3)\}$. Then we have on the support of $\phi_{I}$ that $\left|x_{1}-x_{2}\right| \leq \frac{R}{6}$ (due to the $\operatorname{supp} \chi_{1}$ ) and since $\left|x_{1}\right|>R,\left|x_{2}\right| \geq \frac{5 R}{6}$. Further, due to the supp $\chi_{2}$, we have $\left|x_{1}-x_{3}\right| \geq \frac{R}{12}$. Suppose now, we would choose $P=\{1,2\}$ and $Q=\{3\}$, then, according to (2.1),

$$
U_{P}=\left\{\left(x_{1}, x_{2}, x_{3}\right)|| x_{1}|>\epsilon,| x_{2}|>\epsilon,| x_{1}-x_{3}|>\epsilon,| x_{2}-x_{3} \mid>\epsilon\right\},
$$

for some $\epsilon>0$. But then $\operatorname{supp} \phi_{I} \not \subset U_{P}$, since supp $\phi_{I}$ contains points with $x_{2}=x_{3}$. On the other hand one easily checks that the choice $P=\{1,2,3\}$ is the right one. This example shows that we cannot just choose $P$ to be $\{1\} \cup\{j \leq N \mid(1, j) \in I\}$. On the other hand $P$ cannot be too big: For $N=3, I=\{(1,2)\}, J=\{(1,3),(2,3)\}$ it is easily seen that with $P=\{1,2,3\}, \operatorname{supp} \phi_{I}$ contains points with $x_{3}=0$ and therefore $\operatorname{supp} \phi_{I} \not \subset U_{P}$.

Physically speaking, we divide the $N$ electrons into 2 clusters. The electrons $j$ with $j \in P$ define the 'maximal cluster' of electrons containing the electron 1 . This will be done via an equivalence relation below. Note that the (three-dimensional) variable $x_{P}=\frac{1}{\sqrt{|P|}} \sum_{j \in P} x_{j}$ is (up to a scalar multiple) the centre (centre of mass) of the maximal cluster.

Let $\sim$ denote the equivalence relation on $\{1, \ldots, N\}^{2}$ generated by $I$ and let $P$ denote the equivalence class of 1. 
14S. FOURNAIS, M. AND T. HOFFMANN-OSTENHOF AND T. Ø. SØRENSEN

Explicitly this means that $j \sim k$ if either $j=k$ or there exists a sequence $j_{1}, \ldots, j_{l}$ with $j_{s} \in\{1, \ldots, N\}$ for $1 \leq s \leq l$ and with $j_{s} \neq j_{t}$ for $s \neq t$, such that

(i) $\left(j, j_{1}\right) \in I$ or $\left(j_{1}, j\right) \in I$,

(ii) $\left(j_{s}, j_{s+1}\right) \in I$ or $\left(j_{s+1}, j_{s}\right) \in I$, for $1 \leq s \leq l-1$,

(iii) $\quad\left(j_{l}, k\right) \in I$ or $\left(k, j_{l}\right) \in I$.

Clearly

$$
l \leq N-2 .
$$

Thus $P=\{j \mid j \sim 1\}, Q=\{1, \ldots, N\} \backslash P$. Notice that $P \neq \emptyset$. In order to be able to apply Lemma 2.3, we have to show that $\operatorname{supp} \phi_{I} \subset$ $U_{P}$ (with a suitable choice of $\epsilon$ in the definition of $U_{P}$ ).

Let $j \in P$, then $j \sim 1$ and we can choose a sequence $j_{1}, \ldots, j_{l}$ according to the above. Taking into account $\left|x_{1}\right|>R$ we have

$$
\left|x_{j}\right| \geq R-\left|x_{1}-x_{j}\right| \text {. }
$$

Further, with

$$
\left|x_{1}-x_{j}\right| \leq\left|x_{1}-x_{j_{1}}\right|+\sum_{s=1}^{l-1}\left|x_{j_{s}}-x_{j_{s+1}}\right|+\left|x_{j_{l}}-x_{j}\right|,
$$

the length scale of the cut-off's and (3.1) we obtain that

$$
j \in P \Rightarrow \operatorname{supp} \phi_{I} \subset\left\{\left(x_{1}, \ldots, x_{N}\right) \in \mathbb{R}^{3 N}|| x_{j} \mid>R / 4\right\} .
$$

Remark 3.3. In the case of molecules we get

$$
j \in P \Rightarrow \operatorname{supp} \phi_{I} \subset\left\{\left(x_{1}, \ldots, x_{N}\right) \in \mathbb{R}^{3 N}\left|\min _{1 \leq l \leq L}\right| x_{j}-R_{l} \mid>R / 4\right\} .
$$

Furthermore, suppose $j \in P, k \in Q$, then it is clear that $(j, k) \in J$ or $(k, j) \in J$ (because if $(j, k) \in I$, then $1 \sim j \sim k$ and therefore $k \in P$ ), and therefore:

$j \in P, k \in Q \Rightarrow \operatorname{supp} \phi_{I} \subset\left\{\left(x_{1}, \ldots, x_{N}\right) \in \mathbb{R}^{3 N}|| x_{j}-x_{k} \mid>R /(4 N)\right\}$.

Using (3.2) and (3.3) we see that $\operatorname{supp} \phi_{I} \subset U_{P}$, with $\epsilon=\frac{R}{4 N}$ in the definition (2.1) of $U_{P}$. Hence we get from Lemma 2.3 that $\psi \circ T^{*}$ is (infinitely often) differentiable with respect to the coordinate $x_{P}=$ $\frac{1}{\sqrt{|P|}} \sum_{j \in P} x_{j}$ on the support of $\phi_{I} \circ T^{*}$.

Denote $g_{I}=\psi^{2} \phi_{I}$, and note that all partial derivatives of $\phi_{I}$ are bounded. We get from Lemma 2.3 that

$$
\begin{aligned}
& \partial_{x_{P}}^{\gamma}\left(g_{I} \circ T^{*}\right) \in C^{0,1}\left(\mathbb{R}^{3 N}\right), \\
& \left|\partial_{x_{P}}^{\gamma}\left(g_{I} \circ T^{*}\right)\left(x_{P}, x^{\prime}\right)\right| \leq c_{\gamma} e^{-\lambda\left|\left(x_{P}, x^{\prime}\right)\right|} \text { for all }\left(x_{P}, x^{\prime}\right) \in \mathbb{R}^{3 N} .
\end{aligned}
$$


Now, we are ready to prove the smoothness of the electron density. We calculate (using the notation from (2.2), and (2.7) and (2.8)):

$$
\begin{aligned}
\rho_{I}(x) & =\int_{\mathbb{R}^{3 N-3}} g_{I}\left(x, x_{2}, \ldots, x_{N}\right) d x_{2} \cdots d x_{N} \\
& =\int_{\mathbb{R}^{3 N}} g_{I}\left(x_{1}, \ldots, x_{N}\right) \delta\left(x-x_{1}\right) d x_{1} d x_{2} \cdots d x_{N} \\
& =\int_{\mathbb{R}^{3 N}}\left(g_{I} \circ T^{*}\right)\left(x_{P}, x^{\prime}\right) \delta\left(x-\frac{x_{P}}{\sqrt{N_{1}}}-t_{1}^{*} x^{\prime}\right) d x_{P} d x^{\prime} \\
& =\int_{\mathbb{R}^{3 N-3}}\left(g_{I} \circ T^{*}\right)\left(\sqrt{N_{1}}\left(x-t_{1}^{*} x^{\prime}\right), x^{\prime}\right) d x^{\prime}
\end{aligned}
$$

Using (3.4) and Lebesgue integration theory, we obtain via the chain rule:

$$
\begin{aligned}
\partial_{x}^{\gamma} \rho_{I}(x) & =\partial_{x}^{\gamma}\left(\int\left(g_{I} \circ T^{*}\right)\left(\sqrt{N_{1}}\left(x-t_{1}^{*} x^{\prime}\right), x^{\prime}\right) d x^{\prime}\right) \\
& =\left(\sqrt{N_{1}}\right)^{|\gamma|} \int\left(\partial_{x_{P}}^{\gamma}\left(g_{I} \circ T^{*}\right)\right)\left(\sqrt{N_{1}}\left(x-t_{1}^{*} x^{\prime}\right), x^{\prime}\right) d x^{\prime} .
\end{aligned}
$$

This proves that $\rho$ is smooth away from the nucleus.

The exponential decay of the derivatives of $\rho_{I}$ is a consequence of (3.4). This can be seen by a similar calculation as in (3.5) but in reversed order:

$$
\begin{aligned}
\left|\partial_{x}^{\gamma} \rho_{I}(x)\right| & \leq\left(\sqrt{N_{1}}\right)^{|\gamma|} \int\left|\left(\partial_{x_{P}}^{\gamma}\left(g_{I} \circ T^{*}\right)\right)\left(\sqrt{N_{1}}\left(x-t_{1}^{*} x^{\prime}\right), x^{\prime}\right)\right| d x^{\prime} \\
& \leq c \int e^{-\lambda\left|\left(\sqrt{N_{1}}\left(x-t_{1}^{*} x^{\prime}\right), x^{\prime}\right)\right|} d x^{\prime} \\
& =c \int\left(e^{-\lambda|\mathbf{x}|} \circ T^{*}\right)\left(x_{P}, x^{\prime}\right) \delta\left(x-\frac{x_{P}}{\sqrt{N_{1}}}-t_{1}^{*} x^{\prime}\right) d x_{P} d x^{\prime} \\
& =c \int e^{-\lambda|\mathbf{x}|} \delta\left(x-x_{1}\right) d x_{1} \cdots d x_{N} \\
& =c \int e^{-\lambda\left|\left(x, x_{2}, \ldots, x_{N}\right)\right|} d x_{2} \cdots d x_{N} .
\end{aligned}
$$

Let us write $\left(x, x_{2}, \ldots, x_{N}\right)=(x, z)$. Then for all $\epsilon \in(0,1)$ :

$$
|(x, z)|=(1-\epsilon)|(x, z)|+\epsilon|(x, z)| \geq(1-\epsilon)|x|+\epsilon|z| .
$$

Therefore

$$
\begin{aligned}
\int e^{-\lambda|(x, z)|} d z & \leq e^{-\lambda(1-\epsilon)\left|x_{1}\right|} \int e^{-\lambda \epsilon|z|} d z \\
& =c e^{-\lambda(1-\epsilon)\left|x_{1}\right|} .
\end{aligned}
$$

This verifies inequality (1.10) and finishes the proof of Theorem 1.3. 
16S. FOURNAIS, M. AND T. HOFFMANN-OSTENHOF AND T. Ø. SØRENSEN

Acknowledgement. The first mentioned author wishes to thank ESI and in particular Prof. Jakob Yngvason for hospitality in the spring 2001.

\section{REFERENCES}

[1] Eschrig, H. The fundamentals of density functional theory Teubner-Texte zur Physik. Teubner Verlag, Stuttgart, 1996.

[2] Folland, G. B. Introduction to partial differential equations, second ed. Princeton University Press, Princeton, NJ, 1995.

[3] Fournais, S., Hoffmann-Ostenhof, M., Hoffmann-Ostenhof, T., And ØstergaArd Sørensen, T. Regularity of Electron Molecular Wavefunctions. In preparation.

[4] Gilbarg, D., and Trudinger, N. S. Elliptic Partial Differential Equations of Second Order, second ed. Springer-Verlag, Berlin, 1983.

[5] Hoffmann-Ostenhof, M., Hoffmann-Ostenhof, T., and Østergaard Sørensen, T. Electron Wavefunctions and Densities for Atoms. Ann. Henri Poincaré 2 (2001), 77-100.

[6] Hoffmann-Ostenhof, M., Hoffmann-Ostenhof, T., And Stremnitzer, H. Local Properties of Coulombic Wave Functions. Comm. Math. Phys. 163, 1 (1994), 185-215.

[7] Kato, T. On the Eigenfunctions of Many-Particle Systems in Quantum Mechanics. Comm. Pure Appl. Math. 10 (1957), 151-177.

[8] Kato, T. Perturbation Theory for Linear Operators. Springer-Verlag, BerlinHeidelberg-New York, 1966.

[9] LiEB, E. H. Thomas-fermi and related theories of atoms and molecules. Rev. Modern Phys. 53, 4 (1981), 603-641.

[10] Lieb, E. H., And Simon, B. The Thomas-Fermi theory of Atoms, Molecules and Solids. Advances in Math. 23, 1 (1977), 22-116.

[11] Lieb, E. H., And Simon, B. The Hartree-Fock Theory for Coulomb Systems. Comm. Math. Phys. 53, 3 (1977), 185-194.

[12] Simon, B. Schrödinger semigroups. Bull. Amer. Math. Soc. (N.S.) 7, 3 (1982), $447-526$.

(S. Fournais, T. Hoffmann-Ostenhof, T. Ø. Sørensen) The ERwin Schrödinger International Institute for Mathematical Physics, Boltzmanngasse 9, A-1090 Vienna, Austria

(S. Fournais, present address) Laboratoire de Mathématiques, Université Paris-Sud - BÂt 425, F-91405 Orsay Cedex, France

(M. Hoffmann-Ostenhof) Institut für Mathematik, Strudlhofgasse 4, Universität Wien, A-1090 Vienna, Austria

(T. Hoffmann-Ostenhof) Institut Für Theoretische Chemie, Währingerstrasse 17, Universität Wien, A-1090 Vienna, Austria 
(T. Ø. Sørensen, present address) Department of Mathematical Sciences, Aalborg University, Fredrik Bajers Vej 7G, DK-9220 Aalborg East, DENMARK

E-mail address, S. Fournais: fournais@imf .au.dk

E-mail address, M. Hoffmann-Ostenhof: mhoffman@esi.ac.at

E-mail address, T. Hoffmann-Ostenhof: thoffman@esi.ac . at

E-mail address, T. Ø. Sørensen: sorensen@math.auc.dk 